\title{
Acute and Subacute Oral Toxicity Studies of the Aqueous Extract from the Stem Bark of Ricinodendron Rautanenii Schinz (Euphorbiaceae) in Wistar Rats
}

\author{
Jacquy Joyce Wanche Kojom ${ }^{1}$, Edwige Laure Nguemfo ${ }^{2}$, Marie-Claire Tchamadeu ${ }^{1}$, Calvin Bogning Zangueu ${ }^{1}$, \\ Edwige Laure Lappa ${ }^{1}$, Christelle Stéphanie Sonfack ${ }^{1}$, Alain Bertrand Dongmo ${ }^{* 1}$ \\ ${ }^{1}$ Department of Animal Biology and Physiology, Faculty of Science, P.O Box 24157, University of Douala, Cameroon \\ ${ }^{2}$ Department of Biological Sciences, Faculty of Medicine and Pharmaceutical Science, P.O Box 2701, University of Douala, \\ Cameroon
}

*Correspondence for author: Alain Bertrand Dongmo; alainberd@yahoo.fr

Received 01 July 2020;

Accepted 02 August 2020;

Published 12 August 2020

\begin{abstract}
Ricinodendron rautanenii is a plant, used in traditional medecine to treat fever, eczema, back pain, cancer and stomacal disorders. This work was carried out to evaluate the safety of the aqueous extract from the stem bark of Ricinodendron rautanenii by determining its potential toxicity after acute and subacute administration in Wistar rats. In Acute toxicity assay, the animals received the extract at the single dose of $5000 \mathrm{mg} / \mathrm{Kg}$ and were observed during $48 \mathrm{~h}$ for mortality and any toxicity manifestations. General behavior, adverse effects and mortality were determined for up to 14 days post treatment. In subacute toxicity assay, extract was given orally to rats at doses of $6,20,40$ and $80 \mathrm{mg} / \mathrm{kg} / \mathrm{day}$ for 28 days respectively. Animal body weight, water and food intake, biochemical and hematological parameters were determined. Liver and kidney were examined histologically for any signs of organ damage. No behavioral changes or mortality were recorded in the treated groups. No significant hematological changes were observed in the both sex. The biochemical analysis indicated a significant increase of high density lipoprotein levels in both male and female rats. Histopathological analysis of the liver and kidney did not show any observable cellular damages. The overall finding of this study suggest that, extract of Ricinodendron rautanenii did not cause any death up to a dose of $5000 \mathrm{mg} / \mathrm{kg}$ and can be considered non-toxic. Biochemical and histological studies of the extract did not revealed major signs in subacute toxicity.
\end{abstract}

Keywords: Ricinodendron rautanenii, acute toxicity, subacute toxicity, wistar rats.

\section{Introduction}

The use of traditional and complementary medicine is becoming widely popular both in the developing as well as in the developed nations for treatment of various diseases. Herbal medicine or phytomedicine is recognized as the most common form of alternative medicine ${ }^{[1]}$. The increasing cost, non-availability of modern drugs and limited access to adequate health care have compelled about $80 \%$ of the world population to use herbal prescriptions and natural remedies for primary health care ${ }^{[2]}$. Herbal based medications are often considered to be safe as they are natural and free from side effects ${ }^{[3]}$. Many chemicals that have been shown to be toxic are constituents of plants that form part of the human diet ${ }^{[4]}$. No drug should be used clinically without its clinical trials and toxicity studies ${ }^{[5]}$. Pre-clinical toxicity studies of herbal medicines are essential to identify the safety and the determination of dose level that could be used subsequently ${ }^{[6]}$.
Ricinodendron rautanenii Schniz. (Euphorbiaceae), is a plant widely distributed in subtropical Africa, from the border of northern Namibia and Angola, stretching through southern and western parts of Zambia, Botswana, northwest and central Zimbabwe, Mozambique and South Africa ${ }^{[7,8,9]}$. In traditional medicine, various parts of this plant (roots, leaves, stems and bark) have been commonly used for treatment of stomachache, skin diseases, fever, cancer, measles and sores ${ }^{[8,10,11,12]}$. Ethnobotanical surveys not yet published in some Cameroonian tradipraticians revealed the use of $R$. rautanenii for the treatment of hypertension.

In spite of the wide ethnotherapeutic applications of the plant, there is no literature information related to the safety limits of Ricinodendron rautanenii aqueous extract in traditional medicine. Thus, in the present study, we evaluated the acute and subacute oral toxicity of the aqueous extract from the stem bark of Ricinodendron rautanenii in Wistar rats. 


\section{Materials and methods}

\section{Chemicals reagents}

Enzymatic and colorimetric reagent kits for determination of alanine aminotransferase (ALT), aspartate aminotransferase (AST), creatinine, bilirubin, urea, total cholesterol, HDL-cholesterol, triglyceride and total protein were purchased from SGMItalia. All other chemicals and solvents used throughout this study were purchased from Sigma-Aldrich, Germany.

\section{Animals}

Young female Wistar rats (120-150 g) were used for acute toxicity and adult Wistar rats of both sex (150-180 g) were used for the subacute toxicity. The animals were housed in colony cages, in the animal house of the University of Douala, Cameroon. They received a diet and tap water ad libitum. The experimental protocol was approved by the Institutional Ethic Committee of the University of Douala $\left(\mathrm{N}^{\circ} 2040\right.$ CEI-UDo/06/2019/T) in accordance with the guidelines for the care and use of Laboratory Animal use.

\section{Plant material and extraction}

Ricinodendron rautanenii was collected in Tikar country, West Region-Cameroon in December 2017, identified by M. Tacham, a botanist, and voucher specimen under the number 0028/OU/LBOV has been deposit at the Laboratory of plant organisms. The stem bark of Ricinodendron rautanenii were cut out, dried in the shade, and then crushed. One hundred grams (100 g) of powder were extracted by infusion in 1 liter of boiled distilled water for 20 minutes. After filtration through Whatman filter paper $\mathrm{N}^{\circ} .3$, the filtrate was evaporated at $40 \mathrm{oC}$ using an oven, yielding $1.21 \mathrm{~g}$ powder (w/w: $1.21 \%)$.

\section{Acute assay}

The in vivo acute toxicity studies of Ricinodendron rautanenii stem bark extract were conducted according to the protocols described by the OECD guideline $423^{[13]}$ with a slight modification. The overnight fasted (water ad libitum) female rats was distributed into 3 groups of 3 rats each. A first group (control) received distilled water. The second group received by gavage a unique dose of the extract at $5000 \mathrm{mg} / \mathrm{kg}$ and the third group received the same dose of extract with 48 hours interval. Following administration, animals were observed for signs of toxicity, body weight change and mortality, with attention given during the first 4 hours, and daily thereafter, for a total of 14 days. Neither food nor water was given up to $4 \mathrm{~h}$ after extract administration.

\section{Subacute toxicity}

The experiment was conducted according to the protocol described by OECD Guideline $407^{[14]}$. Sixty rats of both sexes were divised into 6 groups ( $\mathrm{n}=10$ animals/group, 5 males and 5 females). Four different doses of $R$. rautanenii $(6,20,40$ and $80 \mathrm{mg} / \mathrm{kg}$ ) were administered per group, orally (gavage), daily for 28 consecutive days. The control group receive only vehicle (distilled water). Another group (Satellite) received the maximum dose of $80 \mathrm{mg} / \mathrm{kg}$ of plant extract for 28 days and remained untreated for 14 more days. It is important to use a satellite group for the observation of reversibility, persistence, or delayed occurrence of toxic effects related to the administration of the test substance. During the treatment, daily body weight, food and water consumption, and possible signs of toxicity were observed and recorded. At the end of experiment $\left(28^{\text {th }}\right.$ days and $42^{\text {th }}$ days), all the rats were anesthetized under diethyl ether, and their blood samples were collected by retro orbital punction for hematological and biochemical analyses.

\section{Hematological analysis}

A small amount of blood samples collected into sample tubes containing ethylene diaminotetra acetic acid (EDTA). The tubes were shaken gently to mix up the blood with EDTA and prevent clotting. For hematological analysis, red blood cell (RBC), white blood cell (WBC), differential leukocyte cells (lymphocyte, monocyte, granulocyte), hemoglobin concentration (HGB), hematocrit (HCT), mean corpuscular hemoglobin concentration (MCHC), mean corpuscular hemoglobin $(\mathrm{MCH})$, and mean corpuscular volume (MCV) were measured using an automatic hemocyte analyser (Urit, 3000).

\section{Biochemical parameters}

At the end of the treatment, blood samples were collected on anesthetized rats under diethyl ether by retro orbital punction in dry tubes, centrifuged at $3000 \mathrm{rpm}$ for $15 \mathrm{~min}$ to obtain serum which was stored at $-20^{\circ} \mathrm{c}$ for biochemical analysis; aspartate aminotransferase (ASAT), alanine aminotransferase (ALAT), creatinine, bilirubin, urea, total cholesterol, HDL-cholesterol, triglyceride, and protein total (SGMItalia Kits).

The kidneys and liver were identified and carefully dissected out after rinsing in normal saline, representative fragments of kidney and liver, were crushed with Tris- $\mathrm{HCl}$ solution $(20 \%)$ and the supernatant obtained after centrifugation at 3000 rpm for $30 \mathrm{~min}$ was used for the determination of the tissue biochemical parameters (AST, ALT, creatinine, bilirubin and urea).

The biochemical parameters were determined using specific kits (SGMItalia) by measurement of the optical density of the reaction products at the corresponding wave lengths with a spectrophotometer.

\section{Histopathological analysis}

The others representative fragments of kidney and liver, were subsequently fixed in a $10 \%$ solution of buffered formalin ( $\mathrm{pH} 7.4)$ and enclosed in paraffin for histopathological analysis. Fivemicrometer sections were obtained and colored with hematoxylineosin for evaluation under a optical microscope.

\section{Statistical analysis}

The results were expressed as means \pm standard error of the mean (SEM). The comparisons within the experimental groups were performed using One-way analysis of variance test (ANOVA) followed by Dunnett's Multiple Comparison Post test. P values less than 0.05 and 0.01 were considered significant.

\section{Results}

\section{Acute oral toxicity}

General behavior

On the 14 days treatment with the dose $(5000 \mathrm{mg} / \mathrm{kg})$ of $R$. rautanenii stem bark extract, all the female in two groups tests were survived throughout the entire study period. Cage side observations did not record any behavioral changes such as tremor, convulsions, salivation and lethargy during the first four hours after treatment. No change in feces, and body coat aspect, locomotion and reactivity to noise were observed. After $24 \mathrm{~h}$, there was no mortality recorded in treated groups.

\section{Weekly body weight}

The mean of rats' body weight was measured daily for 14 consecutive days. The treatment of rats with plant extract significantly $(p<0.01)$ increased the body weight by $11.73 \%$ and $9.11 \%$ at the 7 and 14 days of treatment respectively, when compared to the group treated with distilled water only (Fig. 1). 


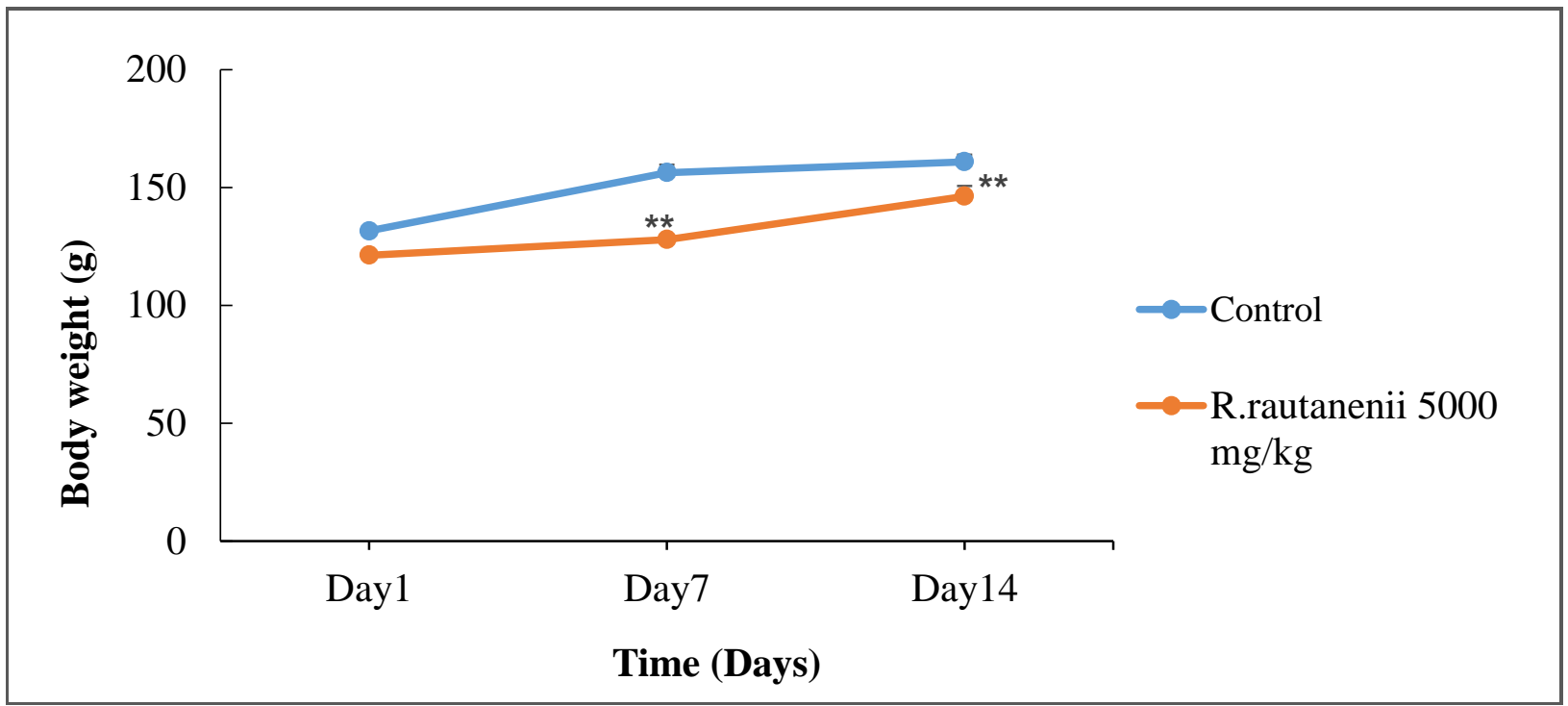

Fig.1: Effect of R. rautanenii extract on body weight in acute toxicity. Each bar represents the means \pm SEM; $\mathbf{n}=5$; **p $<0.01$ : significant difference compared to the control group.

\section{Organ relative weight and histhopathology}

A relative organ weight of rats was determined at the end of acute toxicology test period (14 days). There was no statistical difference in the organ's relative weights (liver, kidney, lungs, heart, aorta and spleen) between treated and control groups (Table 1). No abnormality (color, size, shape and texture) was found in the same organs at necropsy.

Table 1: Effect of extract on relative weight of organs ( $\mathrm{g} / 100 \mathrm{~g}$ of body weight) in acute assay

\begin{tabular}{|l|l|l|l|l|l|l|l|}
\hline & \multicolumn{7}{|l|}{ Relative weight of organs (g/100 g of body weight) } \\
\hline Treatment & Doses & Heart & Kidneys & Lungs & Spleen & Liver & Aorta \\
\hline Control & $10 \mathrm{ml} / \mathrm{kg}$ & $0.30 \pm 0.02$ & $0.90 \pm 0.02$ & $0.82 \pm 0.09$ & $0.31 \pm 0.01$ & $3.14 \pm 0.11$ & $0.05 \pm 0.01$ \\
\hline R. rautanenii & $5000 \mathrm{mg} / \mathrm{kg}$ & $0.35 \pm 0.03$ & $0.90 \pm 0.01$ & $1.20 \pm 0.21$ & $0.32 \pm 0.03$ & $3.81 \pm 0.31$ & $0.10 \pm 0.02$ \\
\hline
\end{tabular}

Data represent the mean $\pm S E M ; n=5 ; R$. rautanenii $=$ Ricinodendron rautanenii.

\section{Subacute oral toxicity}

Body weight food and water consumption

No deaths and unusual changes in behavior or locomotor activity were recorded and no signs of intoxication were observed during the 28 days of treatment. From day 0 to day 28, the increasing of the body weight of rats of both sexes in all the groups was observed. This variation was not significative between the control group and the experimental groups. However, significant variation of the body weigtht was observed on the 7th day of treatment at all the doses tested. This maximum increase reached $12.77 \%$ (p $<0.01$ ) at a dose of $80 \mathrm{mg} / \mathrm{kg}$ compared to control group (Table 2).
The relative organ weights showed no significant differences between treated and control group (Table 3).

The amount of food and water consumed was measured daily from the quantity of food and water supplied and the amount remaining after $24 \mathrm{~h}$.Values for consumption are based on total intake and average relative body weight of the preceding time interval. Water consumption as well as food intake in all groups presented no significant variation between the experimental group and control group.

Table 2: Effect of the aqueous extract of Ricinodendron rautanenii on body weight

\begin{tabular}{|l|l|l|l|l|l|l|l|l|}
\hline & \multicolumn{7}{|c|}{ Body weight (g) } \\
\hline Female & Dose $(\mathbf{m g} / \mathbf{k g})$ & Day0 & Day7 & Day14 & Day 21 & Day 28 & Day 35 & Day 42 \\
\hline Control & - & $141.0 \pm 0.6$ & $163.2 \pm 1.8$ & $164.0 \pm 0.9$ & $188.0 \pm 2.5$ & $188.4 \pm 4.2$ & \\
\hline R. rautanenii & 80 & $163.2 \pm 3.0$ & $175.8 \pm 3.3^{* *}$ & $182.6 \pm 4.6$ & $187.2 \pm 5.9$ & $190.0 \pm 5.4$ & & \\
\hline R. rautanenii & 40 & $165.8 \pm 2.1$ & $188.0 \pm 3.1^{* *}$ & $196.6 \pm 3.5$ & $200.2 \pm 3.4$ & $208.8 \pm 5.0$ & & \\
\hline R. rautanenii & 20 & $156.2 \pm 1.6$ & $180.8 \pm 1.7^{* *}$ & $189.0 \pm 3.0$ & $190.0 \pm 2.9$ & $197.6 \pm 4.2$ & & \\
\hline R. rautanenii & 6 & $148.8 \pm 0.4$ & $173.4 \pm 2.4^{* *}$ & $177.4 \pm 1.6$ & $186.6 \pm 3.3$ & $189.6 \pm 5.0$ & & \\
\hline Satellite & 80 & $156.8 \pm 1.6$ & $181.4 \pm 2.1^{* *}$ & $184.8 \pm 2.9$ & $188.0 \pm 3.6$ & $189.8 \pm 5.5$ & $193.0 \pm 9.5$ & $188.5 \pm 5.6$ \\
\hline Male & & & & & & & & \\
\hline Control & - & $157.0 \pm 4.8$ & $184.2 \pm 5.2$ & $203.0 \pm 8.9$ & $205.6 \pm 7.9$ & $216.0 \pm 7.4$ & & \\
\hline$R$. rautanenii & 80 & $176.0 \pm 2.5$ & $203.4 \pm 4.0$ & $217.6 \pm 5.1$ & $221.4 \pm 6.3$ & $229.4 \pm 5.2$ & & \\
\hline$R$. rautanenii & 40 & $169.6 \pm 4.0$ & $199.8 \pm 2.5$ & $217.8 \pm 3.8$ & $227.4 \pm 4.8$ & $226.4 \pm 5.6$ & & \\
\hline$R$ rautanenii & 20 & $168.6 \pm 8.2$ & $199.4 \pm 7.6$ & $209.2 \pm 4.7$ & $200.4 \pm 5.0$ & $210.4 \pm 9.2$ & & \\
\hline$R$. rautanenii & 6 & $159.4 \pm 4.0$ & $188.0 \pm 4.4$ & $201.2 \pm 4.5$ & $208.0 \pm 4.5$ & $206.8 \pm 4.6$ & & \\
\hline Satellite & 80 & $174.4 \pm 3.6$ & $190.6 \pm 6.2$ & $205.8 \pm 4.2$ & $228.3 \pm 4.6$ & $229.2 \pm 4.6$ & $231.6 \pm 4.3$ & $243.0 \pm 6.5$ \\
\hline
\end{tabular}

Data represent the mean \pm SEM; $n=5$; R. rautanenii $=$ Ricinodendron rautanenii; $* \mathrm{p}<0.05$ and $* * \mathrm{p}<0.01$ : statistically significant compared to the control group. 
Table 3: Effect of the aqueous extract of Ricinodendron rautanenii on relative (g/100g of body weight) organ weight

\begin{tabular}{|c|c|c|c|c|c|c|}
\hline \multicolumn{7}{|c|}{ Relative organ weight (g/100g of body weight) } \\
\hline Female & Doses (mg/kg) & Liver & Kidney & Heart & Lungs & Spleen \\
\hline Control & - & $3.18 \pm 0.15$ & $0.30 \pm 0.01$ & $0.33 \pm 0.01$ & $0.85 \pm 0.09$ & $0.27 \pm 0.01$ \\
\hline$R$. rautanenii & 80 & $3.29 \pm 0.17$ & $0.30 \pm 0.01$ & $0.37 \pm 0.01$ & $0.82 \pm 0.07$ & $0.38 \pm 0.04$ \\
\hline$R$. rautanenii & 40 & $3.09 \pm 0.18$ & $0.26 \pm 0.01$ & $0.33 \pm 0.01$ & $0.90 \pm 0.06$ & $0.35 \pm 0.03$ \\
\hline$R$. rautanenii & 20 & $3.18 \pm 0.12$ & $0.28 \pm 0.01$ & $0.36 \pm 0.02$ & $0.92 \pm 0.13$ & $0.31 \pm 0.05$ \\
\hline$R$. rautanenii & 6 & $2.98 \pm 0.10$ & $0.30 \pm 0.01$ & $0.37 \pm 0.02$ & $0.81 \pm 0.04$ & $0.30 \pm 0.03$ \\
\hline Satellite & 80 & $2.54 \pm 0.05$ & $0.25 \pm 0.01$ & $0.28 \pm 0.00$ & $0.94 \pm 0.05$ & $0.27 \pm 0.03$ \\
\hline \multicolumn{7}{|l|}{ Male } \\
\hline Control & - & $3.03 \pm 0.04$ & $0.29 \pm 0.01$ & $0.27 \pm 0.00$ & $0.76 \pm 0.04$ & $0.31 \pm 0.02$ \\
\hline$R$. rautanenii & 80 & $2.96 \pm 0.11$ & $0.27 \pm 0.01$ & $0.28 \pm 0.01$ & $0.73 \pm 0.02$ & $0.29 \pm 0.03$ \\
\hline$R$. rautanenii & 40 & $2.80 \pm 0.05$ & $0.27 \pm 0.01$ & $0.28 \pm 0.01$ & $0.74 \pm 0.05$ & $0.21 \pm 0.02$ \\
\hline$R$. rautanenii & 20 & $2.86 \pm 0.12$ & $0.29 \pm 0.03$ & $0.30 \pm 0.02$ & $0.79 \pm 0.07$ & $0.27 \pm 0.03$ \\
\hline$R$. rautanenii & 6 & $2.53 \pm 0.06$ & $0.28 \pm 0.01$ & $0.31 \pm 0.02$ & $0.76 \pm 0.05$ & $0.26 \pm 0.02$ \\
\hline Satellite & 80 & $2.31 \pm 0.14$ & $0.22 \pm 0.02$ & $0.25 \pm 0.01$ & $1.01 \pm 0.21$ & $0.24 \pm 0.03$ \\
\hline
\end{tabular}

Data represent the mean $\pm \mathrm{SEM}, \mathrm{n}=5, R$. rautanenii: Ricinodendron rautanenii

Effect of subchronic oral administration of $R$. rautanenii extract on the hematological parameters in rats

There were no treatment-related adverse effects of $R$. rautanenii aqueous extract on hematology parameters in male and female rats (Table 4). The data showed that red blood cell (RBC), hemoglobin concentration (HBG), mean corpuscular hemoglobin concentration (MCHC), mean corpuscular hemoglobin $(\mathrm{MCH})$ and mean corpuscular volume (MCV) differential count levels for control rats were not significantly different from those in treatment groups.

Table 4: Effect of the aqueous extract of Ricinodendron rautanenii on hematological parameters

\begin{tabular}{|c|c|c|c|c|c|c|}
\hline \multicolumn{7}{|l|}{ Female } \\
\hline Parameters & Control & \multicolumn{4}{|l|}{ R. rautanenii } & Satellite \\
\hline Doses & $10 \mathrm{ml} / \mathrm{kg}$ & $80 \mathrm{mg} / \mathrm{kg}$ & $40 \mathrm{mg} / \mathrm{kg}$ & $20 \mathrm{mg} / \mathrm{kg}$ & $6 \mathrm{mg} / \mathrm{kg}$ & $80 \mathrm{mg} / \mathrm{kg}$ \\
\hline $\operatorname{RBC}\left(10^{-6} / \mathrm{mm}^{3}\right)$ & $3.6 \pm 0.4$ & $3.7 \pm 0.2$ & $4.8 \pm 0.5$ & $3.7 \pm 0.3$ & $4.1 \pm 0.5$ & $5.5 \pm 0.4$ \\
\hline WBC $\left(10^{-3} / \mathrm{mm}^{3}\right)$ & $3.8 \pm 0.7$ & $7.7 \pm 0.9$ & $6.2 \pm 1.1$ & $6.9 \pm 1.8$ & $5.1 \pm 0.4$ & $8.2 \pm 0.9$ \\
\hline HBG (g/dl) & $11.0 \pm 1.4$ & $12.0 \pm 0.7$ & $13.4 \pm 0.7$ & $12.8 \pm 0.8$ & $12.5 \pm 1.6$ & $16.1 \pm 1.0$ \\
\hline $\operatorname{HCT~}(\%)$ & $41.5 \pm 1.8$ & $39.1 \pm 2.2$ & $43.0 \pm 1.4$ & $38.9 \pm 1.9$ & $39.4 \pm 4.6$ & $41.8 \pm 3.4$ \\
\hline $\operatorname{MCM}\left(\mu \mathrm{m}^{-3}\right)$ & $64.2 \pm 6.2$ & $60.8 \pm 10.1$ & $78.0 \pm 5.9$ & $52.0 \pm 2.3$ & $77.3 \pm 1.0$ & $84.3 \pm 5.7$ \\
\hline $\mathrm{MCH}(\mathrm{pg})$ & $24.4 \pm 1.3$ & $23.4 \pm 1.9$ & $27.8 \pm 1.4$ & $24.2 \pm 1.9$ & $29.4 \pm 0.7$ & $29.1 \pm 1.1$ \\
\hline $\mathrm{MCHC}(\mathrm{g} / \mathrm{dl})$ & $36.2 \pm 1.7$ & $32.8 \pm 2.2$ & $31.0 \pm 0.6$ & $33.8 \pm 0.7$ & $32.0 \pm 0.9$ & $30.9 \pm 0.8$ \\
\hline $\mathrm{LYM}\left(10^{-3} / \mu \mathrm{l}\right)$ & $1.0 \pm 0.6$ & $3.1 \pm 0.7$ & $3.3 \pm 0.2$ & $3.2 \pm 0.6$ & $3.2 \pm 0.6$ & $1.9 \pm 0.3$ \\
\hline $\mathrm{MON}\left(10^{-3} / \mu \mathrm{l}\right)$ & $0.2 \pm 0.0$ & $0.3 \pm 0.1$ & $0.4 \pm 0.2$ & $0.4 \pm 0.1$ & $0.5 \pm 0.1$ & $0.6 \pm 0.1$ \\
\hline GRA $\left(10^{-3} / \mu \mathrm{l}\right)$ & $3.0 \pm 0.4$ & $3.80 \pm 0.5$ & $4.9 \pm 0.7$ & $3.3 \pm 0.4$ & $3.9 \pm 0.3$ & $4.8 \pm 0.7$ \\
\hline \multicolumn{7}{|l|}{ Male } \\
\hline Parameters & Control & \multicolumn{4}{|l|}{ R. rautanenii } & Satellite \\
\hline Doses & $10 \mathrm{ml} / \mathrm{kg}$ & $80 \mathrm{mg} / \mathrm{kg}$ & $40 \mathrm{mg} / \mathrm{kg}$ & $20 \mathrm{mg} / \mathrm{kg}$ & $6 \mathrm{mg} / \mathrm{kg}$ & $80 \mathrm{mg} / \mathrm{kg}$ \\
\hline $\operatorname{RBC}\left(10^{-6} / \mathrm{mm}^{3}\right)$ & $4.5 \pm 0.8$ & $5.4 \pm 0.5$ & $5.2 \pm 0.6$ & $4.7 \pm 0.7$ & $4.4 \pm 0.8$ & $3.9 \pm 0.4$ \\
\hline WBC $\left(10^{-3} / \mathrm{mm}^{3}\right)$ & $6.0 \pm 1.2$ & $6.5 \pm 1.7$ & $7.3 \pm 1.1$ & $8.0 \pm 0.9$ & $8.3 \pm 1.6$ & $3.5 \pm 0.6$ \\
\hline HBG (g/dl) & $11.8 \pm 1.8$ & $12.6 \pm 0.8$ & $14.5 \pm 0.5$ & $14.5 \pm 0.7$ & $11.4 \pm 1.4$ & $11.6 \pm 0.6$ \\
\hline $\operatorname{HCT}(\%)$ & $44.4 \pm 1.7$ & $41.0 \pm 2.1$ & $45.4 \pm 3.6$ & $44.8 \pm 1.9$ & $46.1 \pm 2.9$ & $48.5 \pm 0.8$ \\
\hline $\operatorname{MCM}\left(\mu \mathrm{m}^{-3}\right)$ & $72.0 \pm 6.3$ & $62.3 \pm 10.1$ & $83.0 \pm 3.7$ & $82.8 \pm 3.3$ & $80.0 \pm 8.4$ & $83.3 \pm 6.3$ \\
\hline $\mathrm{MCH}(\mathrm{pg})$ & $25.0 \pm 1.1$ & $23.4 \pm 3.6$ & $30.0 \pm 0.9$ & $29.9 \pm 1.3$ & $28.1 \pm 2.6$ & $30.0 \pm 1.2$ \\
\hline $\mathrm{MCHC}(\mathrm{g} / \mathrm{dl})$ & $32.8 \pm 0.6$ & $33.1 \pm 2.3$ & $32.01 \pm 0.8$ & $31.0 \pm 0.9$ & $29.3 \pm 1.8$ & $32.5 \pm 1.3$ \\
\hline LYM $\left(10^{-3} / \mu \mathrm{l}\right)$ & $1.26 \pm 0.5$ & $2.3 \pm 1.4$ & $2.56 \pm 0.5$ & $2.52 \pm 0.5$ & $2.3 \pm 0.8$ & $0.5 \pm 0.9$ \\
\hline $\operatorname{MON}\left(10^{-3} / \mu \mathrm{l}\right)$ & $0.36 \pm 0.1$ & $0.4 \pm 0.1$ & $0.53 \pm 0.0$ & $0.43 \pm 0.1$ & $0.5 \pm 0.2$ & $0.4 \pm 0.1$ \\
\hline GRA $\left(10^{-3} / \mu \mathrm{l}\right)$ & $2.44 \pm 0.6$ & $4.1 \pm 0.7$ & $4.92 \pm 0.5$ & $4.80 \pm 0.5$ & $3.9 \pm 0.6$ & $2.4 \pm 0.4$ \\
\hline
\end{tabular}

Data represent the mean $\pm \mathrm{SEM}, \mathrm{n}=5, R$. rautanenii $=$ Ricinodendron rautanenii, ${ }^{* *} \mathrm{p}<0.01$ : statistically significant compared to the control group; RBC : red blood cell, WBC : white blood cell, HBG : hemoglobin, HCT : hematocrit, LYM : lymphocyte, GRA : granulocyte, MCM : mean corpuscular volume, $\mathrm{MCHC}:$ mean corpuscular hemoglobin concentration, $\mathrm{MCH}:$ mean corpuscular hemoglobin ; MON : monocyte

Effect of subchronic oral administration of $R$. rautanenii extract on the biochemical parameters in rats

Table 5 shows a summary of the effects of $R$. rautanenii on the serum biochemical parameters in both sexes. Oral administration of the plant extract at a dose of $6,20,40$ and $80 \mathrm{mg} / \mathrm{kg}$ did not cause significant changes in serum or tissue biochemical parameters such as AST, ALT, bilirubin, creatinine, and urea levels when compared to control group. Moreover total cholesterol, LDL-cholesterol, triglyceride and total protein did not show significant variations. However, the HDL-cholesterol level increased by $20.18 \%$ (p $<0.05)$ and $22.19 \%(\mathrm{p}<0.01)$ in male and female respectively at a dose of $40 \mathrm{mg} / \mathrm{kg}$.

In liver and kidney tissue, no significant variations were recorded for ALAT, ASAT, bilirubin, creatinine and urea 
concentrations of the group treated with extract when compared to

the control group.

Table 5: Effect of the aqueous extract of Ricinodendron rautanenii on biochemical parameters

\begin{tabular}{|c|c|c|c|c|c|c|}
\hline \multicolumn{7}{|l|}{ Female } \\
\hline Parameters & Control & \multicolumn{4}{|l|}{ R. rautanenii } & Satellite \\
\hline Doses & $10 \mathrm{ml} / \mathrm{kg}$ & $80 \mathrm{mg} / \mathrm{kg}$ & $40 \mathrm{mg} / \mathrm{kg}$ & $20 \mathrm{mg} / \mathrm{kg}$ & $6 \mathrm{mg} / \mathrm{kg}$ & $80 \mathrm{mg} / \mathrm{kg}$ \\
\hline CHL (mg/dl) & $84.1 \pm 7.1$ & $76.9 \pm 4.0$ & $81.3 \pm 5.4$ & $81.2 \pm 5.1$ & $83.5 \pm 3.2$ & $73.6 \pm 6.0$ \\
\hline HDL (mg/dl) & $18.2 \pm 0.5$ & $19.0 \pm 1.4$ & $22.8 \pm 0.9^{*}$ & $22.2 \pm 1.4$ & $22.4 \pm 3.2$ & $19.6 \pm 0.7$ \\
\hline LDL (mg/dl) & $45.3 \pm 9.7$ & $43.0 \pm 5.3$ & $39.2 \pm 5.3$ & $42.1 \pm 5.0$ & $43.0 \pm 3.7$ & $37.6 \pm 1.8$ \\
\hline Trig (mg/dl) & $78.5 \pm 5.1$ & $83.7 \pm 1.8$ & $76.6 \pm 9.4$ & $79.2 \pm 6.7$ & $87.4 \pm 6.3$ & $71.6 \pm 6.7$ \\
\hline Prot (mg/dl) & $75.0 \pm 1.8$ & $61.4 \pm 6.7$ & $59.8 \pm 6.0$ & $58.8 \pm 1.7$ & $66.7 \pm 3.7$ & $75.6 \pm 3.3$ \\
\hline Bil (mg/dl) & $0.2 \pm 0.1$ & $0.2 \pm 0.1$ & $0.2 \pm 0.2$ & $0.2 \pm 0.1$ & $0.2 \pm 0.2$ & $0.2 \pm 0.1$ \\
\hline Urea $(\mathrm{mmol} / \mathrm{l})$ & $13.1 \pm 3.0$ & $11.7 \pm 3.6$ & $6.6 \pm 3.0$ & $9.6 \pm 6.6$ & $10.5 \pm 5.4$ & $6.6 \pm 2.3$ \\
\hline Crea (mg/dl) & $1.1 \pm 0.2$ & $0.8 \pm 0.1$ & $0.9 \pm 0.3$ & $0.9 \pm 0.2$ & $1.0 \pm 0.2$ & $1.3 \pm 0.3$ \\
\hline ALT (U/l) & $85.2 \pm 15.2$ & $64.0 \pm 13.7$ & $75.3 \pm 25.6$ & $68.9 \pm 26.0$ & $65.5 \pm 16.8$ & $66.8 \pm 22.1$ \\
\hline AST (U/l) & $140.0 \pm 33.7$ & $136.9 \pm 42.3$ & $118.4 \pm 34.5$ & $127.8 \pm 37.3$ & $111.2 \pm 15.5$ & $88.66 \pm 34.8$ \\
\hline \multicolumn{7}{|l|}{ Male } \\
\hline Parameters & Control & \multicolumn{4}{|l|}{ R. rautanenii } & Satellite \\
\hline Doses & $10 \mathrm{ml} / \mathrm{kg}$ & $80 \mathrm{mg} / \mathrm{kg}$ & $40 \mathrm{mg} / \mathrm{kg}$ & $20 \mathrm{mg} / \mathrm{kg}$ & $6 \mathrm{mg} / \mathrm{kg}$ & $80 \mathrm{mg} / \mathrm{kg}$ \\
\hline CHL (mg/dl) & $85.1 \pm 4.1$ & $84.46 \pm 9.3$ & $93.9 \pm 2.9$ & $84.7 \pm 4.7$ & $95.2 \pm 5.7$ & $84.5 \pm 5.6$ \\
\hline HDL (mg/dl) & $25.0 \pm 0.8$ & $25.2 \pm 1.5$ & $32.1 \pm 2.2^{*}$ & $22.4 \pm 1.1$ & $28.9 \pm 2.1$ & $23.2 \pm 0.3$ \\
\hline LDL (mg/dl) & $49.3 \pm 11.3$ & $48.1 \pm 7.8$ & $45.0 \pm 1.2$ & $47.2 \pm 3.8$ & $46.9 \pm 5.3$ & $51.3 \pm 6.2$ \\
\hline Trig (mg/dl) & $74.0 \pm 4.2$ & $79.6 \pm 11.4$ & $73.2 \pm 2.1$ & $75.8 \pm 4.4$ & $70.7 \pm 3.3$ & $58.8 \pm 5.2$ \\
\hline Prot (mg/dl) & $74.7 \pm 1.9$ & $74.8 \pm 2.4$ & $79.4 \pm 3.1$ & $57.4 \pm 6.3$ & $79.5 \pm 0.4$ & $74.6 \pm 0.8$ \\
\hline Bil (mg/dl) & $0.2 \pm 0.1$ & $0.2 \pm 0.1$ & $0.2 \pm 0.1$ & $0.2 \pm 0.1$ & $0.3 \pm 0.1$ & $0.2 \pm 0.1$ \\
\hline Urea $(\mathrm{mmol} / \mathrm{l})$ & $22.3 \pm 11.2$ & $18.0 \pm 1.5$ & $15.5 \pm 3.1$ & $16.3 \pm 2.2$ & $11.6 \pm 3.0$ & $30.0 \pm 11.3$ \\
\hline Crea (mg/dl) & $1.8 \pm 0.4$ & $1.6 \pm 0.2$ & $1.4 \pm 0.1$ & $1.5 \pm 0.3$ & $1.7 \pm 0.3$ & $1.9 \pm 0.9$ \\
\hline ALT (U/l) & $83.4 \pm 35.9$ & $57.8 \pm 19.1$ & $63.4 \pm 20.2$ & $63.2 \pm 2.4$ & $64.6 \pm 25.7$ & $55.6 \pm 19.7$ \\
\hline AST (U/l) & $141.0 \pm 64.4$ & $113.3 \pm 44.3$ & $111.2 \pm 45.8$ & $136.6 \pm 42.5$ & $118.7 \pm 40.8$ & $112.6 \pm 46.3$ \\
\hline
\end{tabular}

Data represent the mean $\pm \mathrm{SEM}, \mathrm{n}=5, R$. rautanenii= Ricinodendron rautanenii, $* \mathrm{p}<0.05$ : statistically significant compared to the control group; CHL: total cholesterol, HDL : high density lipoprotein, LDL : low density lipoprotein, Pro : total protein, Trig : triglycerides, Bil : bilirubin, Crea : creatinine, ALT: alanine amino transferase, AST : aspartate amino transferase

\section{Histopathological analysis}

Pathological examinations of the tissues indicated that there were no detectable abnormalities and alterations in the microscopic examination of the organs such as liver and kidney. All groups treated with extract showed in representative section of a kidney, normal and intact glomeruli, Bowman's capsule and urinary space
(Fig. 2b, Fig. 2c and Fig. 2d) like to control group (Fig. 2a). Histopathological analysis of the liver in control group (Fig. 3a) and groups tests (Fig. 3b, Fig. 3c and Fig. 3d), showed normal hepatic architecture and normal liver lobular structure with branch of portal vein and hepatic artery, prominent nucleus and wellpreserved cytoplasm. a

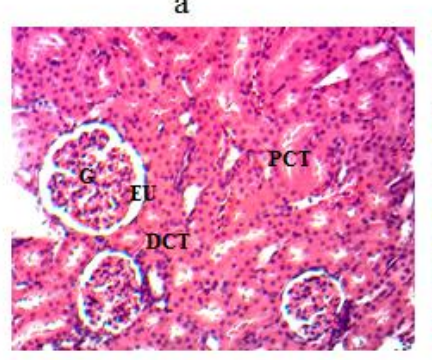

a

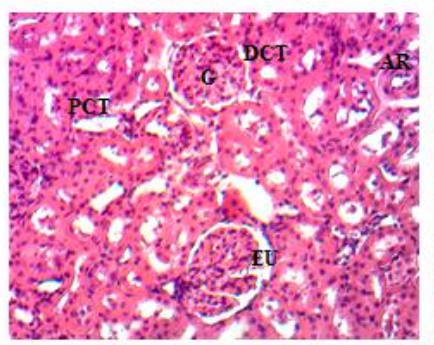

b

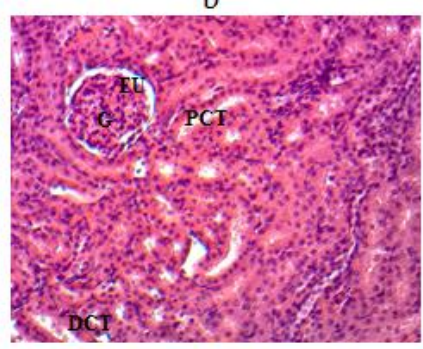

b

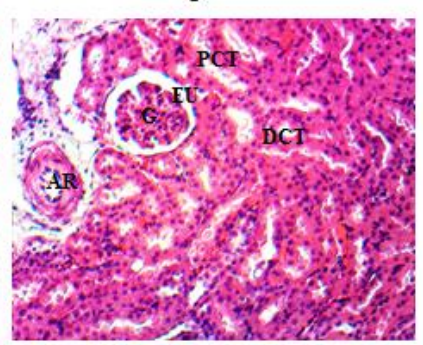

Female

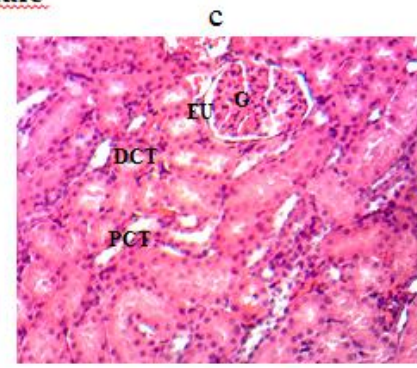

c

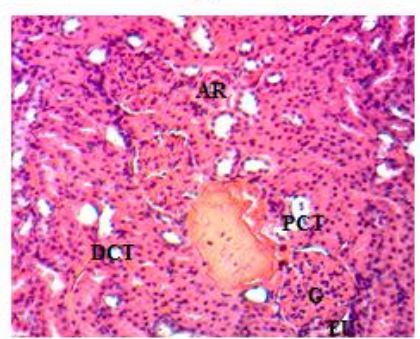

d

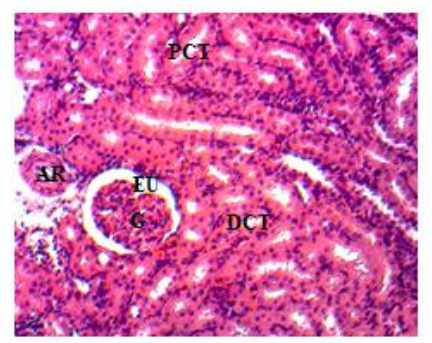

d

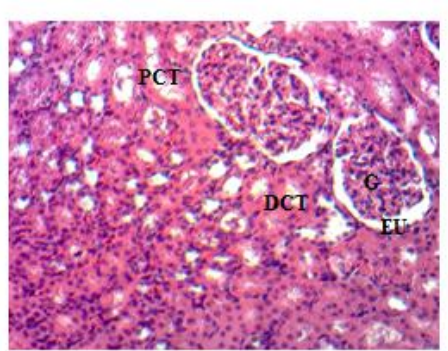

Fig. 2 Histological kidneys cut of rat (HE x 100), G : glomerul, PCT : proximal convoluted tubule, DCT : distal convoluted tubule, EU : urinary space, AR : renal artery, a : control $10 \mathrm{mg} / \mathrm{kg}, \mathrm{b}:$ Ricinodendron rautanenii $6 \mathrm{mg} / \mathrm{kg}, \mathrm{c}$ : Ricinodendron rautanenii $40 \mathrm{mg} / \mathrm{kg}$, d : satellite 80 $\mathrm{mg} / \mathrm{kg}$. 


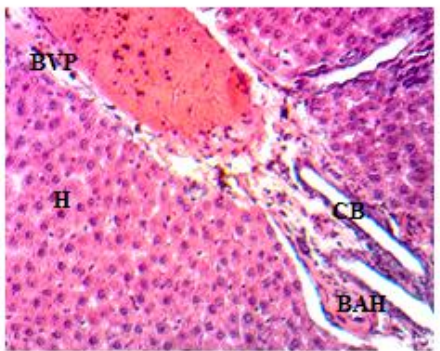

a

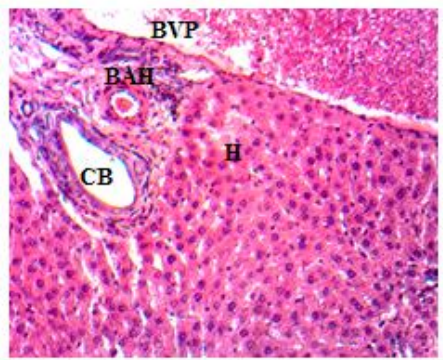

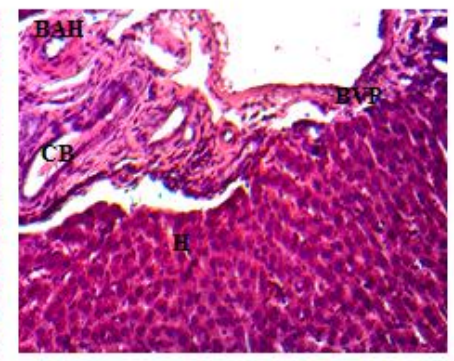

b

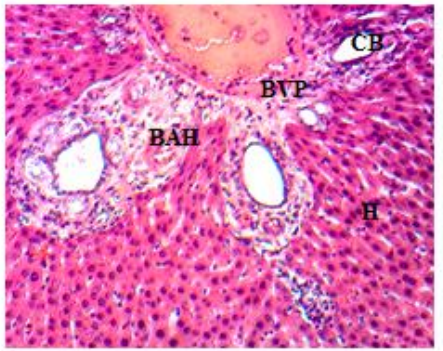

Female

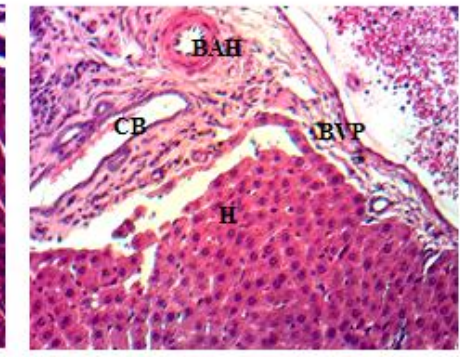

c

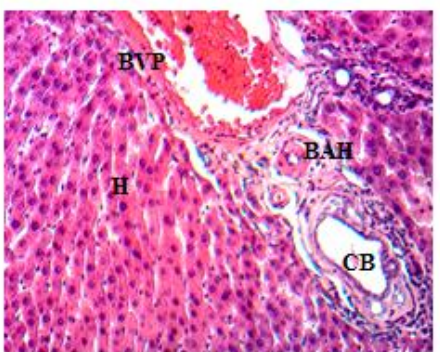

d

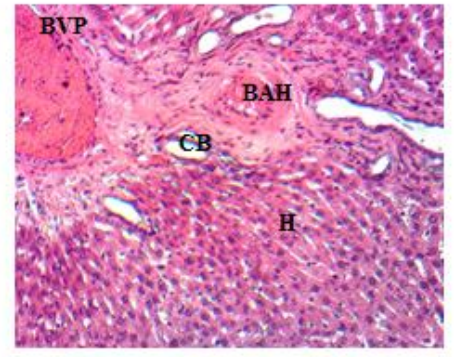

d

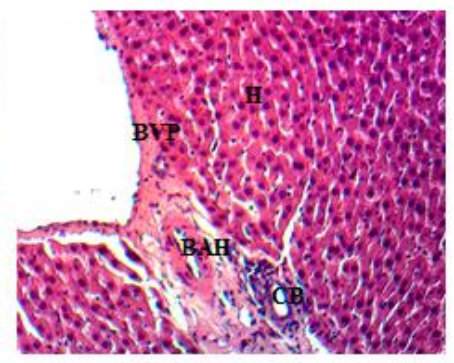

Fig. 3 Histological liver cut of rat (HE x 100), BVP : branch of portal vein, BAH : branch of hepatic artery, CB : bile duct, H : hepatocyte, a : control $10 \mathrm{mg} / \mathrm{kg}$, b : Ricinodendron rautanenii $6 \mathrm{mg} / \mathrm{kg}$, c: Ricinodendron rautanenii $40 \mathrm{mg} / \mathrm{kg}$, d : satellite $80 \mathrm{mg} / \mathrm{kg}$.

\section{Discussion}

The primary aim of toxicological assessment of any herbal medicine is to identify adverse effects and to determine limits of exposure level at which such effects occur. Two important factors which are taken into consideration in evaluating the safety of any herbal drug are the nature and significance of the adverse effect and in addition, the exposure level where the effect is observed. The evaluation of the appropriate effect of Ricinodendron rautanenii for its further development as a therapeutic medicine needed to assess both acute and subacute toxicity for this extract plant.

In acute toxicity assay, no mortalities were observed after the administration of $R$. rautanenii aqueous extract at a dose of $5000 \mathrm{mg} / \mathrm{kg}$ body weight. This showed that the stem bark extract had a negligible level of toxicity when administered orally to rats. Thus, the extract of $R$. rautanenii falls in class 5 (a substance with oral lethal dose $\left(\mathrm{DL}_{50}\right)$ higher than $5000 \mathrm{mg} / \mathrm{kg}$ ), hence considered of non-toxic ${ }^{[13]}$.

The macrocopic examinations of the organs of the animals treated with a single dose $(5000 \mathrm{mg} / \mathrm{kg})$ did not show any changes in color and aspect compared with the control. Also, there were no significant changes in the organs weight of treated animals compared with the control. Subacute studies assess the undesirable effects of continuous or repeated exposure of plant extracts or compounds over a portion of the average life span of experimental animals, such as rodents. Specially, the provide information on target organ toxicity and are designed to identify no observable adverse effect level ${ }^{[15]}$

In this study, subacute toxicity was evaluated in rats at doses of $6,20,40$ and $80 \mathrm{mg} / \mathrm{kg}$ for 28 days. Consideration should be given to an additional satellite group treated with top dose group for observation of reversibility, persistence or delayed occurrence of systemic toxic effects and recovery from toxic effects, for at least 14 days after treatment.

Following the administration of differents doses $(6,20,40$ and $80 \mathrm{mg} / \mathrm{kg}$ ) of aqueous stem bark extract of $R$. rautanenii, no noticeable change in food and water intake was observed. This showed that the oral administration of bark extract did not induce any suppression in appetite and had no deleterious effect on food and water intake. This indicated that the metabolism of carbohydrate, protein, and fat are not affected ${ }^{[16]}$. Moreover, it's known that the monotoring of weight changes and food consumption in studies of pharmacological effects of drugs serve as a sensitive indication of the general health status of animals ${ }^{[17,18]}$. The weight gains were observed in all animals treated with $R$. rautanenii extract. However in 7th days of treatment, a significant increase was observed in female rat at all doses tested when compared to control group. This difference disappeared during the rest of the treatment. No significant body weight changes were observed in male rats. It could be stated that the $R$. rautanenii extract did not interfere with the normal metabolism of animals as corrroborated by the no significant difference from animals in the vehicle. Futhermore, scientific evidence confirmed that increases or decreases in the body weights are accompanied with accumulation of fats and physiological adaptation responses to the plant extracts rather than to the toxic effects of chemicals or drugs that lead to decrease appetite and, hence, lower caloric intake by the animal ${ }^{[19]}$.

Similary, the relative organs weights have been observed in toxicity studies to be a relatively sensitive indicator for particular organs, and thereafter, define toxicity as significant changes observed in those particular organs ${ }^{[20]}$. No significant changes in the weights of the heart, kidney, liver, lungs, spleenn and liver were observed, suggesting that the administration of $R$. rautanenii extract at the subacute oral doses had no effect on the normal growth.

In animal toxicity studies, analysis of blood parameters were important to report any alterations and also to evaluate the relative risk effects on the hematopoietic system when interpreting the findings ${ }^{[21,22]}$. Thus, estimation of blood profile parameters like haemoglobin, total red blood cell (RBC), white blood cell (WBC), packed cell volume (PCV) and platelets count are important indexes in evaluating the physiological and pathological status of man and animals ${ }^{[23]}$. Intake of some toxic plants results in an alteration in the normal level of these parameters ${ }^{[24]}$. Our studies indicated that there were no significant difference between the 
hematological parameters of the rats treated with extract of $R$. rautanenii for 28 days as compare to the control.

Analysis of blood parameters is relevant for risk evaluation, as any changes in the hematological and biochemical systems have a higher predictive value for human toxicity, when data are translated from animal studies ${ }^{[25]}$.

Liver and kidneys function analysis is very important in the toxicity evaluation of drugs and plants extracts as they are both necessary for the survival of an organism ${ }^{[26]}$. Thus, some biomarkers can be used as indicative hepatic functions (ALT, AST, bilirubin), or renal function (creatinine, blood urea nitrogen) $[14,27,28]$.

Many xenobiotics are able to induce some degree of liver injury because of its central role of xenobiotic metabolism, its portal location within the circulation and its anatomical and physiological structure ${ }^{[29]}$. Indeed, analysis of the activities of some basic enzymes (such as ALT, and AST) in the serum or tissue can be used to indirectly assess the integrity of tissues after being exposed to certain pharmacological agents ${ }^{[18,30]}$. These enzymes have a significant intracellular metabolic activity. Their increase in serum might be due to cellular lesions at the hepatic level ${ }^{[31]}$. At same, an increase in tissue or serum bilirubin concentrations occurs as a result of increased breakdown of red blood cells (hemolysis) or liver damage e.g., hepatitis or bile duct obstruction ${ }^{[32]}$. Our results revealed a non-significant increase of ALAT, ASAT and bilirubin concentrations in animals treated with extract plant compared to control group. Moreover, in this study, non-significance difference in serum, tissue urea and creatinine levels was observed in group treated with $R$. rautanenii extract. These results indicate that treatments of rats by the extract did not affect renal and hepatic function.

There were no significant changes in serum lipid profile (total cholesterol, triglyceride, LDL cholesterol) as compared to the control groups. However, a significant increase in HDL-cholesterol levels in both sexes was observed at a dose of $40 \mathrm{mg} / \mathrm{kg}$. These results showed that, the extract had some beneficial effects at the dose of $40 \mathrm{mg} / \mathrm{kg}$ reducing cardiovascular risk factors ${ }^{[33]}$. According to Pocock et al. ${ }^{[34]}$, the proteins are synthetized by the liver. Their increase results from a mobilization of plasma proteins to other tissues when they are lacking, thus suggesting hepatic injury ${ }^{[35]}$. This study shows a non-significant decrease in serum protein level in rats that received the extract plant compared to the control group.

Macroscopic and microscopic examination of organs from animals treated with the extract $(6,20,40$ and $80 \mathrm{mg} / \mathrm{kg})$ and control animals showed normal architecture in liver and kidney, suggesting no detrimental changes and morphological disorder induced by oral administration of the $R$. rautanenii for 28 days of the treatment. Generally, any damage to the parenchymal liver cells results in elevations of both transaminases in the blood ${ }^{[36,37]}$. Thus the no significant increases observed in ALT and AST and bilirubin activities strongly suggest that the subacute administration of $R$. rautanenii extract did not alter the hepatocytes and consequently, the metabolism of rats as observed in the histopathology observations in liver tissue. Any rise in urea and creatinine levels is only observed if there marked damage to functional nephrons ${ }^{[28]}$. Equally, there was also no significant increase in urea and creatinine in the subacute administration of $R$. rautanenii extract when compared to the control group. Histological examinations support the conclusions from biochemical analysis. Therefore, the results recorded in this study demonstrate that the $R$. rautanenii extract did not alter the liver and renal function and further support the non-toxic nature of $R$. rautanenii

\section{Conclusion}

Aqueous extract of $R$. rautanenii was found to be non toxic as it did not cause either any lethality or adverse changes in the general behavior, hematological and biochemical parameters when oral acute and sub-acute toxicities in rats were performed. Chronic toxicity studies are necessary to further the safe use of this plant.

\section{Conflicts of Interest}

None

\section{Acknowledgement}

The grant apparatus support of the Alexander von Humboldt Foundation is gratefully acknowledged. AB Dongmo acknowledges Prof Dr W Vierling (TU-Munich, Germany) for the offer of chemical substances.

\section{References}

[1] Ogbonnia SO, Mbaka GO, Anyika EN, Emordi JE, Nwakakwa N. An evaluation of acute and subchronic toxicities of a Nigerian polyherbal tea remedy. Pakistan Journal of Nutrition, 2011; 10(11):1022-1028.

[2] Wickramasinghe BM. Quality control, screening, toxicity and regulation of herbal drugs. Modern Phytomedicine, 2006; 2:25-57.

[3] Oliveira AKM, Oliveira NA, Resende UM, Martins PFRB. Ethnobotany and traditional medicine of the inhabitants of the Pantanal Negro sub-region and the raizeiros of Miranda and Aquidauna, Mato Grosso do Sul, Brazil. Brazilian Journal of Biology, 2011; 71(1):176-179.

[4] Chambers JC. Disturbed alpine ecosystems: seedling establishment of early and late seral dominant species. Logan, Utah University, 1987; 148p.

[5] Anisuzzaman ASM, Sugimoto N, Sadik G, Gafur MA. Subacute txicitystudy of 5-Hydroxyl-2(Hydroxy-Methyl) 4H-pyran-4 One, isolated from Aspergillus fumigatus. Pakistan Journal of Biological Sciences, 2005; 4(8):1012-1015.

[6] Dipak P, Swati D, Ranjitsinh D, Ramachandran AV. Acute and sub-chronic toxicological evaluation of hydromethanolic extract of Coriandrum sativum L. seeds. Excli Journal, 2012; 11:566-575.

[7] Graz FP. Description and ecology of Schinziophyton rautanenii (Schinz) Radcl.-Sm. in Namibia. Dinteria, 2002; 27:19-35.

[8] Juliani HR, Koroch AR, Simon, JE, Wamulwange, C. Mungongo cold pressed oil (Schinziophyton rautanenii): A new natural product with potential cosmetic applications. Acta Horticulturae, 2007; 756: 407-412.

[9] Maroyi A. Contribution of Schinziophyton rautanenii to sustainable diets, livelihood needs and environmental sustainability in Southern Africa. Sustainability, 2018; 10(3):581.

[10] Cheikhyoussef A, Embashu W. Ethnobotanical knowledge on indigenous fruits in Ohangwena and 
Oshikoto regions in Northern Namibia. Journal of Ethnobiology and Ethnomedicine, 2013; 9(34), 2-12.

[11] Dushimemaria F, Mumbengegwi DR. Proposition of a low cost field assay to determine antiproliferate properties of indigenous plants using Dugesia dorotocephala (brown planaria). Scientific Research and Essays, 2015; 10(4), 144-149.

[12] Elago SN, Tjaveondja LT. A comparative evaluation of the economic contributions and uses of Strychnos cocculoides and Schinziophyton rautanenii fruit trees to poverty alleviation in mile 20 village of Namibia. Agricultural Science and Food Research, 2015; 2(1), 2531.

[13] OECD. OECD guideline for testing of chemicals. Test $\mathrm{N}^{\circ}$ 423: Acute Oral Toxicity - Acute Toxic Class Method. OECD Publishing, 2001; 1-14.

[14] OECD. OECD guidelines for the testing of chemicals. Test $\mathrm{N}^{\circ}$ 407: repeated dose 28-day oral toxicity study in rodents. Paris: OECD Publishing, 2008; 2-8.

[15] NRC. Toxicity testing for assessing environmental agents, Interim Report, National Academies. Press, Washington, DC, USA, 2006.

[16] Klaassen, CD. Casarett and Doull's Toxicology: The Basic Science of Poisons. McGraw-Hill Press, New York, NY, USA, 2001.

[17] Borzelleca JF. A proposed model for safety assessment for macronutrient substitutes. Regulatory Toxicology Pharmacology, 1996; 23(1):15-18.

[18] Hilaly JE, Israili ZH, Lyoussi B. Acute and chronic toxicological studies of Ajuga iva in experimental animals. Journal of Ethnopharmacology, 2004; 91(9):43-50.

[19] Arsad SS, Mohd Esa N, Hamzah H, Othman F. Evaluation of acute, subacute and subchronic oral toxicity of Rhaphidophora decursiva (Roxb.) Schott extract in male Sprague Dawley rats. Journal of Medicinal Plants Research, 2013; 7(41): 3030-3040.

[20] Berenguer-Rivas CA, Castillo AA, Martinez HS, Zapata EP, Hernandez JB, Tasse YM. Acute oral toxicity of Azadirachta indica (Neem Tree). Revista Cubana de Plantas Medicinales, 2013; 18(3):502-507.

[21] Li X, Luo Y, Wang L, Li Y, Shi Y, Cui Y, Xue, M. Acute and subacute toxicity of ethanol extracts from Salvia przewalskii Maxim in rodents. Journal of Ethnopharmacology, 2010; 131(1):110-115.

[22] Rosidah Yam MF, Sadikun A, Ahmad M, Akowuah GA, Asmawi MZ. Toxicology evaluation of standardized methanol extract of Gynura procumbens. Journal of Ethnopharmacology, 2009; 123(2):244-249.

[23] Schlam OW, Jain NC, Carrol EJ. Veterinary Haematology. Lea and Feiber Publication, Philadelphia, $1975 ; 807 \mathrm{p}$.

[24] Ajagbonna OP, Onifade KI, Suleiman U. Haematological and biochemical changes in rats given extract of
Calotropis procera Sokoto. Journal of Veterinary Sciences, 1999; 1(1):36-42.

[25] Olson H, Betton G, Robinson D, Thomas K, Monro A, Kolaja G, Lilly P, Sanders J, Sipes G, Bracken W, Dorato M, Deun KV, Smith P, Berger B, Heller A. Concordance of toxicity of pharmaceuticals in humans and in animals. Regulatoty Toxicology Pharmacology, 2000; 32(1):56-67.

[26] Olorunnisola OS, Bradley G, Afolayan AJ. Acute and sub-chronic toxicity studies of methanolic extract of Tulbaghia violacea rhizomes inWistar rats. African Journal of Biotechnology, 2012; 11(83):14934-14940.

[27] Brandt AP, Oliveira LFS, Fernandes FB, Alba J. Evaluation of prospective hypocholesterolemic effect and preliminary toxicology of crude extract and decoction from Vitex megapotamica (Spreng) Moldenke (V. montevidensis Cham.) in vivo. Revista Brasiliera de Farmacognosia, 2009; 19(2):388-393.

[28] Lameire N, Van Biesen W, Vanholder R. Acute renal failure. The Lancet, 2005; 365(9457):417-430.

[29] Omiecinski CJ, Heuvel PJV, Perdew GH, Peters JM. Xenobiotic metabolism, disposition, and regulation by receptors: From biochemical phenomenon to predictors of major toxicities. Toxicological Sciences, 2011; 120(1):49-75.

[30] Al-Hashem F. Camel's milk protects against aluminum chloride-induced toxicity in the liver and kidney of white albino rats. American Journal of Biochemistry and Biotechnology, 2009; 5(3):98-108.

[31] Obika M, Noguchi H. Diagnosis and evaluation of nonalcoholic fatty liver diseases. Experimental Diabetes Research, 2012; 2012(145754):1-12.

[32] Shatoor AS. Acute and sub-acute toxicity of Crataegus aronia Syn. Azarolus (L.) whole plant aqueous extract in Wistar rats. American Journal of Pharmacology and Toxicology, 2011; 6(2):37-45.

[33] Barnett HA, O'Gara G. Diabetes and the Heart. Clinical Practice Series, Churchill Livingstone: Edinburgh UK, 2003; 7-30p.

[34] Pocock G, Richards CD, Brun JF. Abrégés d'histologie. Masson, Paris, 2004; 638p.

[35] Aniagu SO, Nwinyi FC, Akumka DD, Ajoku GA, Dzarma S, Izebe KS. Toxicity studies in rats fed nature cure bitters. African Journal of Biotechnology, 2002; 4(1):72-78.

[36] Mythilypriya R, Shanthi P, Sachdanandam P. Oral acute and subacute toxicity studies with Kalpaamruthaa, a modified indigenous preparation on rats. Journal of Health Sciences, 2007; 53(4):351-358.

[37] Wasan KM, Najafi S, Wong J, Kwong M. Assessing plasma lipid levels, body weight and hepatic and renal toxicity following chronic oral administration of a water soluble phytostanol compound FM-VP4 to gerbils. Journal of Pharmaceutical Sciences, 2001; 4(3):228-234. 